\title{
The Efficacy of Hyaluronic Acid in the Restoration of Soft Tissue Volume of the Lips and Lower $1 / 3$ of the Face: The Evolution of the Injection Technique
}

\author{
Arnold William Klein ${ }^{1,2}$ \\ ${ }^{1}$ Geffen School of Medicine, University of California, Los Angeles, USA; ${ }^{2}$ Minimally Invasive Aesthetics, LLC, Beverly Hills, USA. \\ E-mail: awkleinmd@aol.com
}

Received August 28 $8^{\text {th }}, 2011$, revised October $5^{\text {th }}, 2011$; accepted November $3^{\text {rd }}, 2011$.

\begin{abstract}
Study Objective: To establish the safety and efficacy of small-gel particle hyaluronic acid (SGP-HA; Restylane ${ }^{\circledR}$, Medicis Aesthetics Inc., Scottsdale, AZ) for lip augmentation. Study Design: This was a Phase 3, prospective, open-label, evaluator-blinded, single-center pilot study of SGP-HA use in lip augmentation. The primary efficacy objectives were to investigate the efficacy of SGP-HA in lip augmentation and to assess subject satisfaction with the procedure 12 weeks after treatment. Secondary efficacy objectives were to validate 3D imaging to measure lip augmentation, identify subject satisfaction at all points in time, and identify the duration of lip augmentation and palpability of SGP-HA in the lips. The primary safety objective was to assess the incidence, duration, and severity of all adverse experiences. Results: All 20 subjects and the treating investigator indicated improvement in the appearance of subjects' lips at weeks 2, 6, and 12. SGP-HA administered for augmentation was well tolerated. Four (20\%) subjects treated with SGP-HA experienced 7 treatment-emergent adverse events (TEAEs). Conclusions: Results of this study show promising efficacy and an absence of safety issues with the use of SGP-HA in lip augmentation.
\end{abstract}

Keywords: Dermal Fillers, GAIS, NASHA, TEAE, Lips, Face, Augmentation

\section{Introduction}

Throughout all races and cultures of humankind, certain features of the female face have attracted males. These consist of a large upper face, small lower face, large round eyes, a small nose, and, last but not least, large full lips [1]. For years, models and actresses have attempted to enhance their lips and lines around the mouth by using lipsticks and covering agents. This is apparent in early screen stars that were able to create the illusion of any lip they desired through the use of make-up. It soon became obvious that the lips were the central cosmetic feature of the lower third of the face. This has even become recognized in the art from the time of the great "Mona Lisa". Art historians have discussed the smile of Mona Lisa for centuries but it is her lips, the only visible female sexual organs, that are responsible manner in which this painting grabs the male eye. The seminal work of Man Ray, the photographer/artist, was a huge painting of a pair of lips gently resting in the countryside. When they are full and well defined, lips impart a sense of youth, health, attractiveness, and sexuality to the bearer. Like many features of the face, as the lips age they become far less attractive. Losing volume, they become thin and flat. Also, due to grinding, wear and inward leaning of the molars and age related osteoporosis of the maxilla, thinning of the distance from the lips to the chin is greatly decreased (Figure 1). As dental height is lost, the face ages such that the ends of the lips hang down, contributing to the marionette lines (Figure 2) [2].

In 1981, FDA approved bovine collagen for injection. In 1984, I reported the use of injectable collagen for lip enhancement. Subsequently, many people felt compelled to define their personal aesthetic goals for lip enhancement. While surgical procedures were developed and discarded, and devices such as Gortex rods (SoftForm ${ }^{\circledR}$ ) also came and went. The use of bovine collagen as a predictable enhancement agent allowed physicians to provide stable lip enhancement which was very natural in appearance [3-5]. Lip augmentations heralded a movement toward the use of temporary fillers for volumetric enhancement instead of simply line elimination [4-10]. 


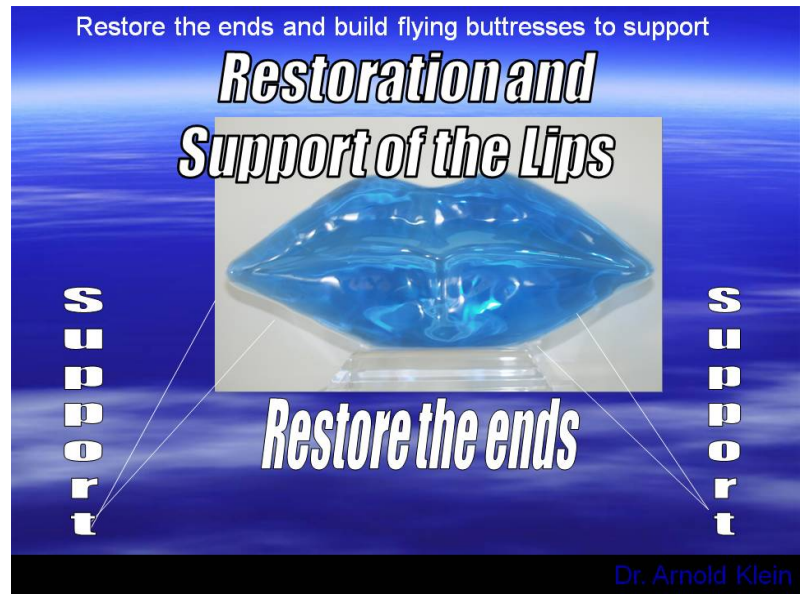

Figure 1. Restoration and support of the lips.

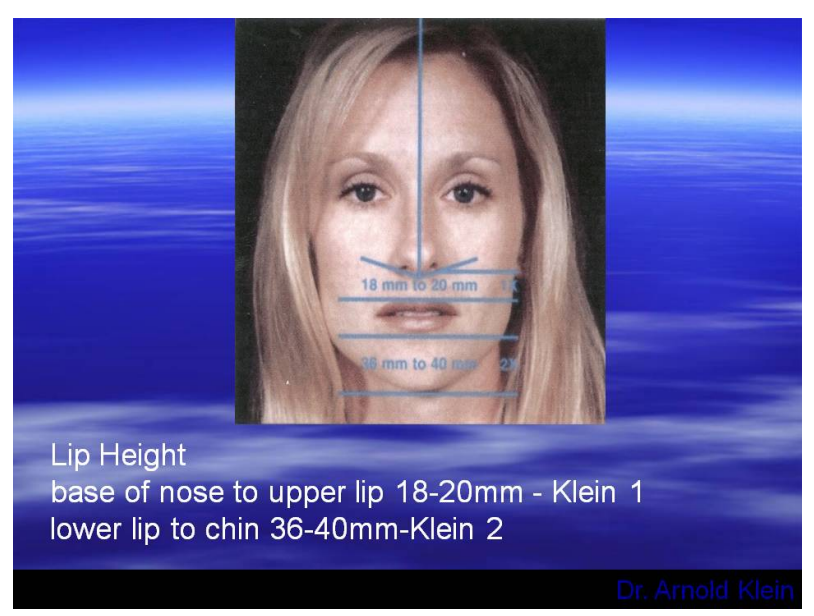

Figure 2. Lip height.

For many years, collagen has been used successfully to eliminate wrinkles and lines in the aging face as well as the ability to restore lost volume [11].

Nevertheless, the dermatologic literature did not contain any information regarding the proper aesthetic characteristics of lips. While women's magazines would present models with voluptuous lips and many celebrities were adored for their lips, no sensible guidelines existed to aid physicians in this area of enhancement [10]. This resulted in aesthetically improper lip enhancement by some physicians through unguided injection procedures. It was this author's opinion and that of others that the injections should to be done in a subtle manner that would prevent the augmentation from being readily apparent. While enhancement of the vermillion border was an approved use of injectable bovine collagen, actual mucosal injection was again a frequent, albeit, off-label use of this agent. Physicians and patients soon began to realize that not only the fullness of the lips but also the shape of the lips needed to be enhanced in order to achieve the proper aesthetic look. Indeed, the areas of the Cupid's bow and philtral pillars were rarely altered in that any distortions in these areas would draw attention to the lip enhancement, and possibly present an artificial appearance and detectable alteration.

Hyaluronic acid products are materials for soft tissue augmentation. Hyaluronic acid is a polysaccharide found naturally in the dermis. Its ability to bind water assists in hydration and provides skin turgor. Unlike collagen, it is identical across all species and is produced by many types of cells. In 2004, hyaluronic acids were FDA approved for use in tissue augmentation $[12,13]$. They are biologically pure with low protein loads [14-17]. Hylaform, Restylane, Perlane, Juvederm, etc can all be used for this purpose. My preferred agents are Restylane, Perlane and Juvederm.

\subsection{The Architecture of the Lip}

Are there any rules we can establish for augmenting the perfect lip? In looking at the aging lip, there are two important areas to observe as stated previously. One is the shape of lips themselves and also in an aging face one must note the importance of the support provided to the lower third of the face by dentition and bone structure [18-21]. These are all features dependent not only on injection of the lips themselves, but also volumetric restoration of the lower third of the face. The lips should be full and well-defined. They should be injected without blunting the edge of the vermilion border of the lips. A physician must also focus on the restoration of the ends of the lips, as well as the building of buttresses at these ends to restore height to the lower third of the face, correcting the labio-mandibular grooves/oral commissures. Neither the dermatologic or plastic surgical literature held the answer for proper lip structure. Instead, the answer was found in the dental literature where many articles have addressed the proper height, size, and location of the lips as produced by dental restoration [18-21]. Remember, proper lip injection cannot be accomplished in five minutes. Restoring the lips, as well as the lower $3 \mathrm{rd}$ of the face, and cheek volume provides so much improvement that patients can, at times, avoid surgery. The injection points are all very important, in that the material should flow towards the middle of the face and pulling the redundant skin with it, therefore preventing the patient from looking too pulled back. When the lips are properly done it should be virtually undetectable; a refreshed and renewed look (Figure 3). Lip enhancement is the number 1 use for injectables. The key is to make the patient look refreshed and natural but undetectable to the casual observer. There is a whole list of permanent 


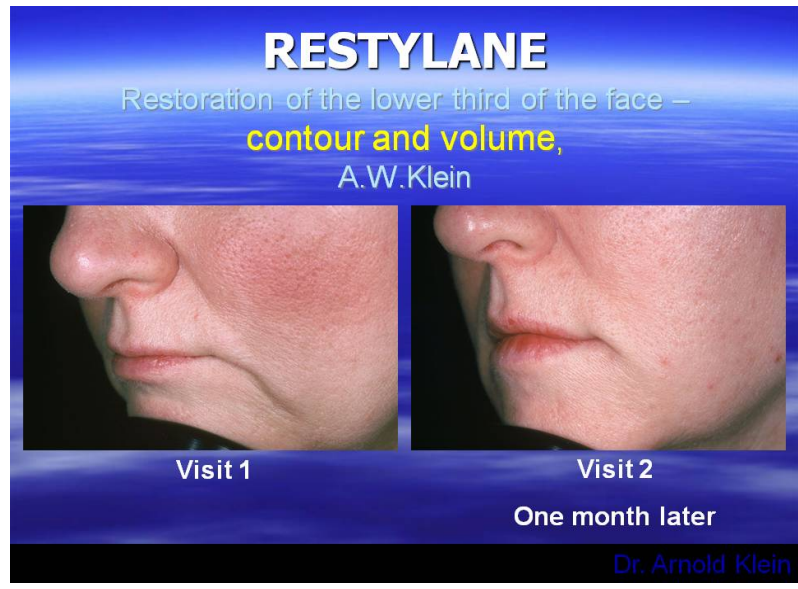

Figure 3. Restoring the lower third of the face.

and so-called semi-permanent injectables with names such as Artefill, Scultptra, Silicone and Radiesse. These should be avoided in that they contain Plexiglas, ground up suture, silicone and bone respectively. None of these agents create collagen but increase volume by causing a foreign body reaction. Often times these reactions cannot be controlled and lumps result.

Small-gel particle and large-gel particle hyaluronic acid (SGP-HA [Restylane] and LGP-HA [Perlane®], respectively, Medicis Aesthetics Inc, Scottsdale, AZ) were developed as an alternative to bovine collagens for soft tissue augmentation. HA, a polysaccharide, occurs naturally in the human dermis. It is less likely than bovine collagen to be associated with immunogenicity and hypersensitivity if free of source protein. Furthermore, studies have indicated that it offers a more lasting outcome [23]. SGP-HA is a gel composed of small particles of HA molecules stabilized by cross-linking. This stabilized gel is more resistant to enzymatic degradation than bovine collagen, and can bind significant amounts of water. These properties might contribute to its greater longevity in cosmetic procedures [24].

To achieve accurate correction and an aesthetically pleasing result with a minimum of side effects, it is essential to employ the proper injection technique. Unfortunately there is a paucity of literature concerning this. The author has perfected the anterior, or serial puncture, technique, which allows the gentle, controlled forward flow of SGP-HA in a mid-dermal level, resulting in a minimum of lumps or bruises [22,24-26].

Lip augmentation does not simply involve increasing the size of lips; it also involves addressing the restoration of the architectural features of the lip (Figures 1, 2). A full understanding of these features is necessary to create the "perfect lip". Lip augmentation involves restoration of the lower third of the face to position the lips at their proper distance from the nose to the upper lip as well as lower lip to the chin [2] as illustrated in Figure 3. We define the distance from the upper lip to the nose Klein 1 and the distance from the lower lip to the chin as Klein 2. SGP-HA is approved for use in treatment of moderate to severe wrinkles The current approval of this agent for lip augmentation is based on this and an additional study. It is well known the author pioneered the use of this agent in the lips [1] and there have been anecdotal reports of its use elsewhere for this purpose [1,8-10]. This pilot study in 20 patients was undertaken to investigate the safety and efficacy of SGP-HA for lip augmentation.

\subsection{Study Objectives}

The co-primary efficacy objectives of this study were to determine whether SGP-HA was effective in lip augmentation and to assess subject satisfaction with the procedure 12 weeks after treatment. Secondary efficacy objectives were to identify subject satisfaction at all points in time, and identify the likely duration of effect of both lip augmentation and intended palpability of SGP-HA in the lip.

The primary safety objective was to assess the incidence, duration, and severity of all systemic and local adverse experiences.

\section{Methods}

Study design: This was a Phase 3, prospective, open- label, evaluator-blinded, single-center pilot study of the use of SGP-HA in lip augmentation. SGP-HA was administered at the baseline treatment session, with efficacy assessments conducted on day 3 , and then at 2, 6, 12, and 24 weeks after initial treatment. The study was conducted with the approval of the Institutional Review Board and was monitored according to good clinical practices. Written informed consent was obtained from each patient prior to entry into the study. The first subject entered the study on May 23, and the last subject completed the study on January 23. Additionally, because of the subtlety of correct lip augmentation a record of Klein Distance 1 and 2 were recorded.

Selection of study population: Healthy adult subjects 18 to 75 years of age were included in the study. Subjects had previously decided to seek lip augmentation and abstain from any other facial plastic surgical or cosmetic procedure for the first 12 weeks of the study. Subjects may have undergone facial cosmetic procedures outside the area of assessment either before or contemporaneously with augmentation.

Subjects who had known hypersensitivity to SGP-HA, or those with existing disease on entry that may have resulted in changes in facial contour or edema during the 
course of the study (e.g., inflammation, infection, facial psoriasis, herpes zoster, cancer, pre-cancer, actinic keratosis), or any tissue augmentation therapy in the preceding 8 months were excluded from the study. Additional exclusion criteria included the use of platelet-inhibiting agents or other anticoagulants in a relevant period before study entry; incomplete recovery from or plans to have a facial procedure; a history of severe allergies manifested by anaphylaxis; use of any tissue-augmenting therapy or aesthetic facial surgery below the level of the lower orbital rim within 6 months before the study; known allergies or hypersensitivity reactions to local topical anesthetics; cancerous or precancerous lesions in the treatment area; use of investigational drugs or other medical devices under investigation within 30 days before entry; and any condition that the investigator believed made the subject unable to complete the study per protocol.

Treatment: Subjects who met all of the inclusion criteria and none of the exclusion criteria were enrolled in the study and 2D and 3D images were obtained before treatment. Each subject was then treated with SGP-HA for optimal lip augmentation (i.e., the lip volume enhancement identified before treatment as cosmetically desirable by the investigator and subject). A diary was given to each subject for daily recording of any local (injection site) and systemic adverse events (AEs) during the first 2 weeks of the study. There was 1 day of treatment with follow-up visits through 24 weeks for safety and efficacy assessments.

Lip augmentation was performed by the investigator, including anesthesia, aseptic precautions, and any indicated pretreatment, such as the administration of botulinum toxin above the lower orbital rim. SGP-HA was administered by injection of the lips. SGP-HA was supplied in presterilized $1.0 \mathrm{ml}$ syringes and a sterilized 30 G.0.5-inch needle. The amount of dermal filler used to provide optimal correction of the lips was recorded and identified for each subject. Per protocol, repeat SGP-HA injections were not permitted for correction on follow-up visits.

Subjects returned for a follow-up visit on day 3 for an AE review, a check of diary completion, and an assessment of product palpability by both the subject and investigator.

The $2 \mathrm{D}$ and $3 \mathrm{D}$ photography was performed at baseline and at 2, 6, 12, and 24 weeks. The 2D images were assessed by a blinded evaluator, the investigator, and the subject. Each viewer identified whether the newer image demonstrated lip augmentation compared with the baseline image.

Efficacy variables: The 2 primary efficacy variables were the proportion of study subjects with identifiable lip augmentation at 12 weeks as measured blindly from 2D photographs or by greater than $20 \%$ increase in Klein line 2 plus Global Aesthetic Improvement Scale (GAIS) ratings at 12 weeks.

1) blind lip evaluation at 12 weeks or

2) greater than $20 \%$ increase in Klein line 2 plus.

\section{Gais}

Subjects considered their satisfaction with the filler placement using the GAIS rating (improved, same, worse) at each efficacy follow-up $(2,6,12$, and 24 weeks). Subjects also assessed lip palpability at 72 hours and 2 weeks.

Secondary efficacy variables included the investigator's assessment at each efficacy follow-up $(2,6,12$, and 24 weeks) compared with the anticipated GAIS rating (improved, same, worse) originally projected for that follow-up visit.

Three-dimensional imaging was conducted for each patient for up to 24 weeks, or until the imaging no longer showed an improvement in the patient's lip appearance over time according to the investigator.

Selection of study population: Healthy adult subjects 18 to 75 years old were included in the study. Subjects had previously decided to seek lip augmentation and abstain from further treatment during the course of the study.

The duration of lip augmentation was assessed by both subject examination during the initial 2-week period and by the investigator at 72 hours, and at 2, 6, 12, and 24 weeks using GAIS. The actual palpability of the product in the lip as compared with the anticipated palpability at each follow-up was assessed by the treating investigator throughout the study.

Safety variables: Safety assessments included all systemic and local AEs at each post-treatment study visit (72 hours and 2, 6, 12, and 24 weeks) and patient observations through use of the daily diary during the 2 weeks following treatment.

Clinical assessment: Subjects returned the diary at the 2 -week visit. Live assessments of lip augmentation were performed at 2, 6, 12, and 24 weeks and compared with baseline, and at each of these times 2D and 3D images were obtained and separate live GAIS ratings of the lips were performed by the subject, and investigator. The blinded investigator only evaluated the physical width of the lips themselves at the central point of the mouth which proved to be a poor measurement of augmentation success.

\section{Results}

Demographics: A total of 20 subjects were enrolled in the study. The mean age of study subjects was 52.8 years, 
and most were female $(90 \%)$ and white $(85 \%)$.

Efficacy: All subjects and the treating investigator indicated lip improvement for all study subjects at weeks 2 , 6 , and 12. At 12 weeks, all subjects rated themselves as improved on their GAIS assessments $(\mathrm{P}<0.001 ; 90 \%$ CI $0.84,1.00)$; the investigator's GAIS assessment also rated all patients as improved at this time $(\mathrm{P}<0.001$; $90 \%$ CI $0.85,1.00)$. The blinded evaluator judged lip improvement in all subjects at week 2 , which decreased to $89 \%, 37 \%$, and $29 \%$ at weeks 6,12 , and 24 , respectively. At week 24, investigators indicated an improvement in $84 \%$ of subjects and $74 \%$ of subject self-assessments indicated an improvement. The median duration of effect was 92 days for the blinded evaluator and 183 days for subjects. Nevertheless, a $20 \%$ improvement in the Klein 2 line was maintained for $84 \%$ for at least 90 days after 1 injection. Since this was an objective measurement it proved superior to the blinded investigator's global assessment.

Intended palpability of the product was detected by the investigator through week 2 of the study. SGP-HA was palpable in at least $1 \mathrm{lip}$ in as much as $85 \%$ of subjects at 72 hours after administration, which decreased to $35 \%$ or less by week 2. SGP-HA was not palpable to the investigator at any time after 2 weeks following injection. All instances were determined by the investigator to be the intended feel of the product.

Statistically significant changes from baseline through week 24 were demonstrated in the Canfield 3D system in some measurements, and some lip measurements were highly correlated with others measured by the Canfield 2D and 3D systems. Specifically the decreased Klein 1 line and increased Klein 2 line were maintained for 24 weeks.

Safety: Seven TEAEs were experienced by $4(20 \%)$ subjects treated with SGP-HA. The majority (5/7) were not considered related to treatment. Two subjects had 1 event each of mild bruising considered to be caused by the injection procedure, but these were anticipated events resulting from the administration route and resolved within 2 weeks.

There were 2 serious AEs during the study. One subject had an event of thyroid neoplasm that resulted in the subject's death; one subject was hospitalized for cellulitis of the left lower extremity, refractory to antibiotic therapy, which did not result in the subject's discontinuation from the study. Both of these AEs were considered unrelated to the study treatment.

\section{Discussion}

This 20-subject pilot study evaluated a single open-label treatment of SGP-HA in both lip volume augmentation and architecture. Study subjects were uniformly satisfied with their lip improvement; all subjects indicated improvement in the appearance of their lips through week 12 , and $74 \%$ maintained their assessment of improvement through week 24 . Similarly, $100 \%$ of treating investigator assessments indicated improvement through week 12 , and $84 \%$ indicated improvement through week 24. The latter evaluation did not correlate well with the blinded evaluator's assessments, in which $37 \%$ of subjects were rated as improved at 12 weeks. However, the blinded evaluator did not personally see any subject at any post-treatment visit and performed all baseline and follow-up comparisons using photographs, a protocol deviation that resulted in very inaccurate ratings of lip augmentation due to the blinded evaluator's unfamiliarity with the architectural features of lip augmentation such as improvement in Klein 1 and 2 lines.

Intended palpability of the product was detected by the investigator through week 2 . Product palpation was reported in $90 \%$ of subject diaries. It should be noted that subjects were instructed to report anything they could feel inside the mouth as mass formation, whereas the investigator could discern the palpability of SGP-HA to be the intended feel of the product. Most subjects assessed the mass formation to be tolerable and indicated duration of less than 2 weeks. Therefore, patients considered bruising an $\mathrm{AE}$ but recorded product palpation as lumps in the diary.

In this study, SGP-HA treatment was well tolerated. Four $(20 \%)$ of the subjects treated with SGP-HA experienced 7 TEAEs. The majority (5/7) of these were considered unrelated to treatment. Overall, as assessed by subject diaries, most AEs were tolerable and resolved within 2 weeks.

Because the current study was open label, there was no control arm for comparison purposes. Therefore, the intensity and duration of specific events collected in subject diaries were compared with data from an earlier study involving SGP-HA in the treatment of nasolabial folds. These parameters (bruising, redness, swelling, pain, tenderness, itching, and mass formation) were also compared at 72 hours and 2 weeks after treatment as identified by the investigator. A large majority of subjects in the lip enhancement study assessed symptoms as tolerable in their diaries. At 2 weeks and thereafter, no symptoms were identified by the investigator in the lip study, compared with 21 events identified in the nasolabial fold study. This suggests strongly the superiority of the injection technique used by the investigator in this lip protocol.

In conclusion, SGP-HA has been shown to be effective and to lack safety issues when used for lip augmentation. Although the small sample size might limit any clinically 
meaningful conclusion from the data, the results of this study have been corroborated by the investigator's use of SGP-HA for lip augmentation in over 1000 patients.

\section{REFERENCES}

[1] N. Etcoff, "Survival of the Prettiest: The Science of Beauty," Doubleday, New York, 1999.

[2] K. M. Robertson, W. K. Dyer and W. K. Dyer II, "The Use of Fillers in the Aging Patient," Facial Plastic Surgery, Vol. 12, No. 3, 1996, pp. 293-301. doi:10.1055/s-0028-1082420

[3] A. W. Klein, F. D. Brandt, D. A. Grekin and H. Mittleman, "Collagen Implants for Lip Augmentation," Journal of Dermatologic Surgery and Oncology, 1990.

[4] A. W. Klein, "Injectable Collagen: A Tutorial," In: L. M. Dzubow, Ed., Cosmetic Dermatologic Surgery, Lippincott-Raven, Philadelphia, 1998, pp. 19-34.

[5] A. W. Klein, "Injectable Bovine Collagen,” In: A. W. Klein, Ed., Tissue Augmentation in Clinical Practice, Marcel Dekker, New York, 1998, pp. 125-144.

[6] J. O. Guerrissi, "Surgical Treatment of the Senile Upper Lip," Plastic and Reconstructive Surgery, Vol. 106, No. 4, 2000, pp. 938-940. doi:10.1097/00006534-200009040-00036

[7] S. S. Ergun, D. I. Cek, H. Baloglu, Z. Algun and H. Onay, "Why Is Lip Augmentation with Autologous Fat Injection Less Effective in the Vermilion Border?" Aesthetic Plastic Surgery, Vol. 25, No. 5, 2001, pp. 350-352. doi:10.1007/s00266-001-0020-Z

[8] S. A. Castor, W. C. To and F. A. Papay, "Lip Augmentation with Allo Derma Cellular Allogenic Dermal Graft and Fat Auto Graft: A Comparison with Autologous Fat Injection Alone," Aesthetic Plastic Surgery, Vol. 23, No. 3, 1999, pp. 218-223.

[9] C. Le Louarn, "[Botulinum Toxin and Facial Wrinkles: A New Injection Procedure] Toxine Botulique et Rides Facials: Une Nouvelle Procedure d'Injection," Annales de Chirurgie Plastique et Esthetique, Vol. 43, No. 5, 1998, pp. 526-533.

[10] G. Felman, "Direct Upper-Lip Lifting: A Safe Procedure," Aesthetic Plastic Surgery, Vol. 17, No. 4, 1993, pp. 291-295. doi:10.1007/BF00437101

[11] G. Flageul and L. Halimi, "Injectable Collagen: An Evaluation after 10 Year's Use as a Complement of Plastic Surgery. Le Collagene Injectable: Bilan après 10 Ans d'Utilisation en Complement de la Chirurgie Esthetique," Annales de Chirurgie Plastique et Esthetique, Vol. 39, No. 6, 1994, pp. 765-771.

[12] B. P. Maloney, "Cosmetic Surgery of the Lips," Facial

\section{List of Abbreviations}

GAIS Global Aesthetic Improvement Scale
Plastic Surgery, Vol. 12, No. 3, 1996, pp. 265-278.

[13] R. S. Narins, F. Brandt, J. Leyden, Z. P. Lorenc, M. Rubin and S. Smith, "A Randomized, Double-Blind, Multicenter Comparison of the Efficacy and Tolerability of Restylane versus Zyplast for the Correction of Nasolabial Folds," Dermatologic Surgery, Vol. 29, No. 6, 2003, pp. 588-595.

[14] P. Friedman, et al., "Safety Data of Injectable Nonanimal Stabilized Hyaluronic Acid Gel for Soft Tissue Augmentation," Dermatologic Surgery, Vol. 28, No. 6, 2002, pp. 491-494. doi:10.1046/j.1524-4725.2002.01251.x

[15] S. Schanz, et al., "Arterial Embolization Caused by Injection of Hyaluronic Acid (Restylane)," British Journal of Dermatology, Vol. 146, No. 5, 2002, pp. 928-929.

[16] D. D. Waddell, "Acute Reaction to Intra-Articular Hylan G-F," Journal of Bone and Joint Surgery, Vol. 85. No. 8, 2003, p. 1620.

[17] A. Oswald and A. Gachter, "Acute Reaction to Intra-Articular Hylan G-F for Treatment of Gonarthrosis Onset," Schweizerische Rundschau für Medizin Praxis, Vol. 89. No. 46, 2000, pp. 1929-1931.

[18] R. Rifkin, "Restoring Vertical Dimension to Improve Function, Facial Harmony, and Dental Esthetics," Contemporary Esthetics and Restorative Practice, 2004, pp. 40-46.

[19] E. A. McLaren and R. Rifkin, "Macroesthetics: Facial and Dentofacial Analysis," Journal of the Canadian Dental Association, Vol. 30. No. 11, 2002, pp. 839-846.

[20] J. Niamtu, "Cosmetic Oral and Maxillofacial Surgery: The Frame for Cosmetic Dentistry," Dentistry Today, Vol. 20, No. 4, 2001 pp. 88-91.

[21] J. T. Cheng, S. W. Perkins and M. M. Hamilton, "Perioral Rejuvenation," Facial Plastic Surgery, Vol. 8, No. 2, 2000, pp. 223-323.

[22] M. L. Elson, “Anesthesia for Lip Augmentation," Dermatologic Surgery, Vol. 23, No. 5, 1997, p. 405.

[23] A. W. Klein and B. W. Ayers, "Lip Augmentation," In: S. J. Aston, Ed., Aesthetic Plastic Surgery, Elsevier BV, Amsterdam, 2009, pp. 855-860.

[24] A. W. Klein, "Soft Tissue Augmentation 2006: Filler Fantasy," Dermatologic Therapy, Vol. 19, No. 3, 2006, pp. 129-133. doi:10.1111/j.1529-8019.2006.00066.x

[25] A. A. Jacono, "A New Classification of Lip Zones to Customize Injectable Lip Augmentation," Archives of Facial Plastic Surgery, Vol. 10, No. 1, 2008, pp. 25-29. doi:10.1001/archfaci.10.1.25

[26] J. Carruthers, A. W. Klein, A. Carruthers, R. G. Glogau and D. Canfield, "Safety and Efficacy of Nonanimal Stabilized Hyaluronic Acid for Improvement of Mouth Corners," Dermatologic Surgery, Vol. 31, No. 3, 2005, pp. 276-280. doi:10.1111/j.1524-4725.2005.31073

LGP-HA Large-gel particle hyaluronic acid

SGP-HA Small-gel particle hyaluronic acid

TEAE Treatment-emergent adverse event 